\title{
Erratum to: A state-by-state comparison of middle school science standards on evolution in the United States
}

Bertha Vazquez* ${ }^{*}$

\section{Erratum to: Evo Edu Outreach (2017) 10:5 DOI 10.1186/s12052-017-0066-2}

In the original publication [1] are the values for North Carolina and Nebraska incorrect in Table 2. Below you will find the correct versions.

Incorrect version:

\begin{tabular}{|c|c|c|c|c|c|c|c|}
\hline \multirow[t]{3}{*}{ State } & \multirow{2}{*}{$\begin{array}{l}\text { Category } 1 \\
\text { Is the term } \\
\text { "evolution" used? }\end{array}$} & \multirow{2}{*}{$\begin{array}{l}\text { Category } 2 \\
\text { Is the concept } \\
\text { of evolution } \\
\text { clearly defined? }\end{array}$} & \multirow{2}{*}{$\begin{array}{l}\text { Category } 3 \\
\text { Multiple lines of evidence for } \\
\text { evolution are presented (fossils, } \\
\text { homologous structures, genetics, } \\
\text { artificial selection, etc.) }\end{array}$} & \multirow[b]{2}{*}{$\begin{array}{l}\text { Category } 4 \\
\text { Natural selection } \\
\text { Mentioned } \\
1 \text { point } \\
\text { Defined } \\
1 \text { point }\end{array}$} & \multirow[b]{2}{*}{$\begin{array}{l}\text { Category } 5 \\
\text { Adaptation } \\
\text { Mentioned } \\
1 \text { point } \\
\text { Defined } \\
1 \text { point }\end{array}$} & \multirow{2}{*}{$\begin{array}{l}\text { Total } \\
\text { points }\end{array}$} & \multirow{2}{*}{$\begin{array}{l}\text { Letter } \\
\text { grade }\end{array}$} \\
\hline & & & & & & & \\
\hline & Max $1 \mathrm{pt}$ & Max 1 pts & Max 4 pts & Max 2 pts & Max 2 pts & 10 & \\
\hline NE & 0 & 0 & 1 & 1 & 1 & 3 & $\mathrm{~F}$ \\
\hline NC & 1 & 1 & 4 & 2 & 2 & 7 & 10 \\
\hline \multicolumn{8}{|c|}{ Correct version: } \\
\hline \multirow[t]{3}{*}{ State } & Category 1 & Category 2 & Category 3 & Category 4 & Category 5 & Total & Letter \\
\hline & $\begin{array}{l}\text { Is the term } \\
\text { "evolution" used? }\end{array}$ & $\begin{array}{l}\text { Is the concept } \\
\text { of evolution } \\
\text { clearly defined? }\end{array}$ & $\begin{array}{l}\text { Multiple lines of evidence for } \\
\text { evolution are presented (fossils, } \\
\text { homologous structures, genetics, } \\
\text { artificial selection, etc.) }\end{array}$ & $\begin{array}{l}\text { Natural selection } \\
\text { Mentioned } \\
1 \text { point } \\
\text { Defined } \\
1 \text { point }\end{array}$ & $\begin{array}{l}\text { Adaptation } \\
\text { Mentioned } \\
1 \text { point } \\
\text { Defined } \\
1 \text { point }\end{array}$ & points & grade \\
\hline & Max $1 \mathrm{pt}$ & Max 1 pts & Max 4 pts & Max 2 pts & Max 2 pts & 10 & \\
\hline NE & 1 & 0 & 1 & 0 & 1 & 3 & $\mathrm{~F}$ \\
\hline NC & 1 & 1 & 4 & 2 & 2 & 10 & $A$ \\
\hline
\end{tabular}

The online version of the original article can be found under doi:10.1186/s12052-017-0066-2.

\section{Publisher's Note}

Springer Nature remains neutral with regard to jurisdictional claims in published maps and institutional affiliations.

*Correspondence: bertha@richarddawkins.net

Richard Dawkins Foundation for Reason and Science, Washington, USA
Published online: 11 September 2017

\section{Reference}

1. Vazquez B. A state-by-state comparison of middle school science standards on evolution in the United States. Evo Edu Outreach. 2017;10:5. doi:10.1186/s12052-017-0066-2.

\section{Springer Open}

C The Author(s) 2017. This article is distributed under the terms of the Creative Commons Attribution 4.0 International License (http://creativecommons.org/licenses/by/4.0/), which permits unrestricted use, distribution, and reproduction in any medium, provided you give appropriate credit to the original author(s) and the source, provide a link to the Creative Commons license, and indicate if changes were made. 